\title{
Abuso Sexual Infantil (ASI): Comprensiones y Representaciones Clínicas desde las prácticas de Salud Mental
}

\section{Sexual Child Abuse: Understanding and Representing Clinical Practices within Mental Health Practices.}

\author{
Irene Magaña \\ Carlos Ramírez \\ Luciano Menéndez \\ Universidad de Santiago de Chile, USACH Chile
}

Rec (25 de Octubre de 2013) Acept (01 de Abril de 2014)

\section{Resumen}

\begin{abstract}
Este artículo presenta una investigación, realizada en dos etapas, que estudia al Abuso Sexual Infantil (ASI) como fenómeno clínico, enmarcada en sus expresiones representacionales, conceptuales y culturales. Interroga a profesionales de instituciones que trabajan con abuso, trazando un itinerario analítico comprensivo de abordajes tanto clínicos, como de políticas de intervención desarrolladas.

Metodológicamente realizamos un estudio observacional, analítico y longitudinal ambispectivo, con diseño cualitativo, aplicado en dos cohortes de tiempo (2005-2006 y 2011-2012), para profundizar el conocimiento de comprensiones y estrategias clínicas que profesional e institucionalmente han abordado al ASI.

Sus resultados destacan: sobrejudicialización y sobrepatologización del ASI, necesidad de profundizar el trabajo interdisciplinario, dificultades del abordaje individual de reparación y prevención, desasosiego y malestar subjetivo de los profesionales, presencia social y mantención de situaciones de abuso con patrones de género predominantemente autoritarios, y devastador daño psíquico de víctimas directas e indirectas. Palabras clave: Abuso Sexual Infantil (ASI), judicialización, patologización, género y desasosiego profesional.
\end{abstract}

\section{Abstract}

This study investigates Sexual Child Abuse (SCA) in its clinical context, including its representational, conceptual, and cultural expressions. Working with professionals of institutions, following an analytic yet understanding method, this study traces their approaches at the clinical level as well as intervention policies. Methodologically, the investigation is observational, analytic, and longitudinal, and qualitative design study, including two temporal cohorts (2005-2006 and 2011-2012). These methods were chosen to acquire better knowledge regarding the strategies used in clinics.

Relevant results include: excessive tendencies to treat the matter as a juridical case and as pathology; the need for interdisciplinary work; the difficulty of repairing and preventing; the presence of discontent and unease in professionals related to their work; social relations in the presence of SCA of an authoritarian type; and the devastating psychic damage, both direct and indirect, in its victims.

Keywords: Sexual Child Abuse (SCA), judicial system, pathologies, gender, professional discontent.

Correspondencia: Irene Magaña Frade, Directora del Doctorado en Psicología, Escuela de Psicología, Universidad de Santiago de Chile, USACH. E-mail irene.magana@usach.cl. Ecuador 3650, tercer piso. Estación Central, Santiago, Chile. Fono (56-2) 27184367. 


\section{Introducción}

El Abuso Sexual Infantil (ASI) es sin duda una de las formas de maltrato más devastadoras infringidas por los adultos en contra de los menores, y por tanto, el de mayores consecuencias generalizadas en la persona del niño/a (Gonçalves y Ferreira, 2002; Habigzang y Caminha, 2004).

En particular, el abuso sexual se suscita vulnerando la cualidad vincular interna a la familia; y provocando un fenómeno sociocultural que rompe con todo el imaginario instituido en torno a la función propia del cuidado adulto y a la institución familiar y social, transgrediendo los límites del poder y la confianza, depositada en la función de protección del niño/a por parte de un adulto, que es considerada fundante y articuladora de la vida psicológica infantil (Renvoize, 1982).

Desde una perspectiva más amplia, el ASI es una forma de violencia que involucra desde las dimensiones más intimas del sujeto a aquellas "macro" relativas a las relaciones de género y poder que tocan tanto las esferas de lo público como de lo privado, e impactan en las transformaciones culturales y psicosociales del individuo en la sociedad contemporánea, y aún su comprensión permanece restringida al campo de lo delictual, en parte, "por las exigencias jurídicas que desconsideran las dimensiones psicológicas y social es que configuran tal fenómeno" (Habizang, Koller, Azen Azevedo y Machado, 2005, p. 347), configurando un desencuentro en la articulación entre el campo del derecho y la subjetividad (Herrero, 2009).

En los últimos años este tema se ha hecho presente en el debate público nacional, adquiriendo matices disciplinares diversos, donde la psicología clínica se ha posicionado con sesgos teóricos y terapéuticos que no necesariamente reflejan una comprensión global y acabada del fenómeno como problema que involucra más que a una patología individual, a un fenómeno social polivalente.

Muchas veces, en el discurso mediático predominan sus tintes morbosos y patologizantes, convirtiendo al ASI en una de las formas de abuso y violencia de más difícil comprensión, y dificultando así la posibilidad de intervención y tratamiento por parte de las instituciones que se dedican a abordar el tema.

En los últimos años, la visibilización de las situaciones de ASI ha instalado el debate y su concientización en la opinión pública, surgiendo agrupaciones que apelan a un sistema de protección y de sanción más severo, que se corresponde en cierta medida con la intensidad con que es subjetivado el daño, constituyéndose estas reivindicaciones en referentes para la agenda social que, convirtiendo a sus voceros en representantes de un sentir mayor, instalan lecturas que alimentan ciertos estereotipos de la construcción de género, que lejos de ayudar, confunden aún más el problema.

Si tenemos la intención de resignificar el tema, se hace crucial dilucidar el cómo los organismos y profesionales abordan el abuso sexual infantil, y cómo representan y sistematizan el cuerpo de conocimientos que intervienen en él. En este sentido, interesa investigar sobre las complejas relaciones entre las políticas públicas y jurídicas, las intervenciones particulares de las instituciones abocadas al problema, los diversos actores involucrados, las víctimas y los abusadores, y por supuesto, la mirada de los profesionales de la psicología que intervienen en el ASI (Habizang, Koller, Azen Azevedo y Machado, 2005). Concretamente en Chile, se ha constatado escasez de estudios que investiguen desde la perspectiva de los propios profesionales (Gómez y Haz, 2008) y que aborden, desde esta mirada, los procesos de intervención y reparación del ASI con modelos comprensivos que incluyan niños y familias en sus contextos psicosociales y culturales (Gómez, Muñoz y Haz, 2007).

Es en este contexto, el presente artículo refiere un estudio que tiene por objetivo explorar el quehacer de diversas instituciones que trabajan en el ámbito del abuso infantil en la Región Metropolitana, específicamente desde las percepciones que los profesionales tienen desde sus propias intervenciones, realizado en dos cohortes de tiempo, entre los años 2005-2006 y 2011-2012, para recabar información sobre las miradas profesionales al fenómeno del ASI y cómo ellas se han desarrollado, en vistas de la comprensión y representación de este complejo problema, en estos últimos años.

\section{Antecedentes contextuales}

Si bien en la mayoría de las sociedades el ASI se observa como un fenómeno altamente patológico y antinatura, en él se ejercen otros tipos de violencia, ya sea en la familia (involucrando a cónyuges o hijos), en instituciones, o como actos de individuos aislados, los que generalmente son tolerados y aceptados como parte del funcionamiento social y de la cultura. Se instala entonces la violencia, ya sea invisibilizada como métodos de educación, o silenciada en los intersticios proporcionados por el artificio de la división entre lo público y lo privado, o por la ideología imperante acerca del control adulto y de las familias como espacios protectores o lugares de afecto y cuidado. Así, muchas veces, la violencia transforma estos lugares en uno de terror y miedo, donde protector y victimario difuminan sus fronteras (Fresno y Spencer, 2011), produciendo efectos 
traumáticos y devastadores, ocultos en un discurso que mistifica y falsea dicha condición, y que permite que la violencia y el abuso puedan ser vistos incluso asociados a una función de protección (UNICEF, 2006). Además, la violencia intrafamiliar puede ser comprendida desde el concepto de recursividad que, como señala Lira (en Morales y Lira, 2000), se relaciona con otros niveles de contexto, creándose así nexos entre violencia instalada en las estructuras sociales con reproducción de patrones violentos al interior de la familia. En esta realidad social se inserta la infancia chilena, teniendo estadísticamente una presencia de un $30.9 \%$ de la población total, con una distribución por sexo de un $50.9 \%$ masculino y un $49.1 \%$ femenino (INE en ACHNU, 2010).

De otra parte, los estudios de género relacionados con la violencia sexual registran que los actos de violencia representan el máximo ejercicio de poder de hombres sobre mujeres, y que este dominio no se debe al deseo sexual, sino a la relación de las construcciones del género, donde se atribuyen al hombre la agresividad y la separación de afectos de la relación familiar y sexual. Además, aún asistimos a tiempos en que la estructura patriarcal es la que organiza nuestra sociedad, asumiendo una división de roles sociales que determina la subordinación por sexo y por edad. De tal manera que cuando el infante es niña, su obediencia al adulto hombre es doblemente esperada en función de estos dos aspectos. Considerando las estadísticas, vemos que un $82.9 \%$ de los casos denunciados por abuso sexual infantil posiciona a las niñas como principales víctimas (UNICEF, 2006), lo que corrobora esta existencia de abuso diferenciado por género. Por tanto, el ASI, más que desde las teorías sexuales, encuentra su coherencia comprensiva y/o explicativa desde la manifestación del ejercicio del poder, la violencia y la dominación masculina y patriarcal.

Si revisamos los factores de vulnerabilidad del ASI, el género, la edad y la familia juegan un papel fundamental. Así, las niñas tendrían más probabilidades de sufrir abusos sexuales (AIHW, 2012), y la edad de mayor vulnerabilidad identificada sería entre los 7 y 12 años. Estudios connotan que los aspectos familiares funcionarían en la doble línea de factores protectores pero también de riesgo, siendo el abuso de drogas parental, la presencia de hombres no paternos dentro del hogar, la violencia doméstica y el estilo de crianza punitivo, los aspectos que estarían asociados a un mayor riesgo de abuso en la niña/o (Nelson et al, 2002) y que toda conducta en la que un menor es utilizado como objeto sexual por parte de otra persona, con la que mantiene una relación de desigualdad (edad o poder y/u otros), es abuso sexual infantil (Lameiras, 2002).
No obstante estas explicaciones, clínicamente se sigue pensando en relacionar el ASI al concepto de pedofilia, considerando a éste como un diagnóstico clínico entendido a manera de una parafilia, que consiste en la excitación o el placer sexual, derivados de actividades o fantasías sexuales repetidas o exclusivas con menores pre-púberes (Echeburúa y Guerricaechevarría, 2005). Desde la clínica, difícilmente se considera que la pedofilia también posee matices con implicancias a nivel psicosocial, que plantean la necesidad de abordar en profundidad otras tramas de significado, subyacentes al fenómeno, y que revelan connotaciones que sólo pueden ser comprendidas desde las tramas sociales de género y poder en que se incluyen los niños/as y sus familias.

\section{Metodología}

A fin de entender tanto las opiniones como las diferentes prácticas de los profesionales de equipos de salud que intervienen en el enfrentamiento del ASI, se realiza una investigación exploratoria de tipo cualitativo, cuyo diseño, llamado de tendencia, permite analizar longitudinalmente cohortes diferentes para una misma realidad social, en su situación habitual (Canela, Cea, Galindo y Valilla, 2010), y esperar un resultado de interés en relación a un mismo factor de exposición. Así, se realiza un estudio de tipo analítico, observacional y ambispectivo (Martínez, 2006) de dos cohortes, transversales y dinámicas, de profesionales que trabajaban o trabajan en centros que atienden problemáticas de ASI. Para ambas cohortes se seleccionaron muestras distintas y comparables, representativas de su punto temporal.

En cada una de las cohortes (2005/2006 y 2011/2012) se tomaron a ocho profesionales de distintos centros de salud familiar de la Región Metropolitana, algunos públicos y otros privados pero todos con atención en temas de violencia intrafamiliar (VIF) y abuso sexual infantil (ASI) en sus programas de intervención. En todos ellos, también, hay tres líneas de trabajo fundamentales: ayuda terapéutica, preventiva y capacitación.

\section{Procedimiento}

Con ambas cohortes se utilizó el mismo procedimiento de trabajo: se realizaron entrevistas estructuradas según un mismo propósito y una misma guía temática previamente definida, de manera de hacer posible tanto una categorización transversal (con inferencias inductivas y deductivas) como una integración total de la información obtenida en ambas cohortes. Así, en base al análisis axial de las entrevistas, se 
establecieron una serie de categorías articuladas según los objetivos perseguidos por esta investigación.

Todas las entrevistas se realizaron en las instituciones de trabajo de los informantes.

Además, observando convenciones normativas, en ambas cohortes se aplicaron las entrevistas luego del acuerdo previo e informado de cada uno de los actores participantes del estudio.

\section{Resultados}

La triangulación de la información dio lugar a una sistematización abierta, que se organizó en torno a la comparación de las dos cohortes estudiadas, obteniendo una categorización común para ambas.

1. Modelo teórico sistémico y sus alcances: En ambas cohortes, los modelos de intervención mencionados por los profesionales son inscritos en la perspectiva sistémica, en tanto se reconocen las problemáticas propias del ASI como internas a la organización social y familiar, cuyos elementos se ven mutuamente influenciados. En ese sentido, se declara que la VIF y el ASI son abordados desde tres sistemas interrelacionados: el macrosistema, referido al orden social, cultural y económico, el mesosistema referido a las redes sociales y el microsistema familiar.

Así, a juicio de ambas cohortes, el modelo sistémico sólo permitiría una aproximación capaz de integrar en lo técnico la intervención terapéutica. No se reportan inscripciones teórico/conceptuales de otras intervenciones psicosociales ni terapéuticas más integradoras que las determinadas por la casuística de cada situación. Además, el modelo sistémico se circunscribe al sistema familiar y no a los contextos más amplios.

Cabe señalar que, en la segunda cohorte, se menciona como superpuesta al modelo sistémico la presencia de las dimensiones del género y del poder, produciendo, entonces, una cierta integración de éstas al esquema de intervención psicosocial y terapéutico.

1.a. ASI, daño psíquico y relaciones de violencia: Las/ os profesionales de ambas cohortes definen al ASI como una forma de ejercer violencia por medio de la sexualidad. Paradojalmente, este abuso es tratado no como si fuese violencia, si no como patología, que en su contexto más macro se delimita en lo que se comprende como el fenómeno de la pedofilia y la pederastia, que consideran fundamentalmente al ASI como el abuso sexual de un adulto/a a un menor en términos de objeto y de acción. Para el abuso incestuoso, lo particular es el abuso en el seno de la familia, generando una dinámica de relación alterada para las/los involucradas/ os, donde lo relevante es la triangulación desparentalizada de la víctima, hija que es convertida en amante, madre invisibilizada y padre amante de la hija, inscribiendo en la niña o niño un devastador daño psíquico que, por lo demás, reaparece siempre, aunque a veces de manera subrepticia, deslizándose como síntoma en el entramado familiar.

En ambas cohortes aparecen significados sobre la pedofilia asociados a una parafilia, y acentuando su carácter transgresor como una patología sexual, en el caso de la pederastia. Se señala ya sea como producto de historia de abuso o de características de personalidad psico o sociopáticas (pedofilia) u otro tipo de acciones patológicas (pederastia). Además se hace una referencia parcial (sólo segunda cohorte) al concepto de poder.

1.b. Inscripción del ASI como ejercicio abusivo y/o violento del poder: El ASI es enmarcado en el ejercicio y el abuso de poder de un adulto hacia un/a niño/a, para alcanzar ciertos objetivos que se manifiestan, además de en el plano psicológico, físico y económico-social, en el plano sexual. En la primera cohorte se inscribe al ASI en la violencia como asunto privado, en donde participan un sinnúmero de factores del sistema familiar. En la segunda cohorte, sin embargo, aun en el ámbito de lo privado, se acentúa su aspecto relacional y se lo reubica, por tanto, en el plano interindividual, en un contexto donde se pone en relieve, además, la comprensión de la dinámica relacional patológica implícita en el fenómeno. Por último, en ambas cohortes existe consenso en considerar al ASI como un aspecto del ejercicio y de la violencia intrafamiliar.

2. Relevancia social del género: En ambas cohortes, la categoría del género se instala como condición social imperante, asociada a las características que asume en nuestro país. Machismo como práctica social hegemónica, con su concomitante ejercicio abusivo del poder, y por otro lado, una construcción de subjetividad femenina predominantemente relacionada a lo doméstico, a la satisfacción de necesidades de los otros por sobre las propias (predominancia del cuidado) $\mathrm{y}$, sobre todo, una relación problemática que aún la sociedad chilena tiene con la sexualidad. Condiciones subyacentes a la violencia sexual en general y de ASI en particular.

2.a. Dinámicas femenino/masculino: En ambas cohortes se conceptúa al abuso siguiendo una relación lineal desde la caracterización individual a la social. Sin embargo, este comienzo en el plano individual resulta maximizado respecto a las consideraciones que desde lo social merece la construcción de género, con sus implicancias en las situaciones de ASI. Lo masculino, por un lado, sitúa al hombre como dueño de la sexualidad de la mujer y se relaciona a mitos 
de mayor necesidad sexual e irracionalidad y dependencia respecto de sus dinámicas pasionales. Lo femenino ubica la sexualidad de las mujeres en el plano de la satisfacción del otro, y de la dependencia y objetivación de su deseo por parte de lo masculino.

2.b. La función materna: Si bien la función de la madre es mencionada de manera relevante en ambas cohortes, aparece de forma contradictoria y confusa, incluyendo situaciones que van desde el polo de develamiento del secreto al de mantención de éste.

Todos los expertos tienden a destacar que, en el caso del incesto, la madre juega el rol de víctima y cómplice a la vez, lo que hace aún más difícil su plena participación en los procesos de apertura del secreto familiar y la futura reparación.

3. Reparación/Salud mental: En ambas cohortes aparece la importancia institucional de la denuncia como parte del proceso reparatorio, aunque no siempre es abordada de forma directa y explícita. Se destacada el papel de la acogida por parte de las instituciones judiciales. Además, en este contexto se mencionan a las malas prácticas institucionales como parte importante de la re-victimización y otras dificultades de la intervención.

En otra perspectiva, los profesionales remarcan la relevancia del peso de las políticas jurídicas y la legalidad que emana de ellas, en el sentido de hegemonizar la conceptualización y definición de los casos y dar forma a las líneas de intervención sobre la VIF y ASI, marcando así los derroteros del abordaje terapéutico y el tipo de discurso reparador que se elabora desde lo psicológico.

3.a. Relevancia de la familia en el ASI: Ambas cohortes opinan que el ASI, así como otras problemáticas relacionadas a transgresiones de derechos sexuales y reproductivos (violación, acoso sexual y otros), se relacionan en forma directa con otras patologías femeninas, afectando tanto al equilibrio emocional como psicológico de la mujer. Además, en la mayor parte de casos de ASI están asociadas otras vulnerabilidades como son: carencias vinculares, falta de redes sociales, patologías crónicas como la depresión, estrés, ansiedad, ideación suicida y otras. Estas consideraciones apuntan a señalar la ausencia de trabajo terapéutico dirigido hacia la dinámica familiar y el total desentendimiento de trabajo relacionado con el abusador, que generalmente se produce en este tipo de contextos familiares.

3.b. La complementareidad factor riesgo/factor protector: En ambas cohortes se reconoce la presencia de factores protectores y de riego asociados al ASI, aunque con algunos matices. En la cohorte 2005-2006 se identifican y resaltan: violencia social, exceso de autoritarismo y patriarcalismo al interior de la familia, hacinamiento, madres poco protectoras, ausencia de figuras significativas para el/la menor, roles de género excesivamente rígidos y tradicionales.

Por su parte, la cohorte 2011-2012 coincide en señalar a la violencia social en su conjunto como principal factor implicado en el ASI, enfatizando en los problemas sociales que se involucran en el fenómeno, como condicionantes y facilitadores de la violencia como problema transversal a la totalidad del orden social. No obstante, a pesar de esta mirada, la familia emerge como el referente fundamental a comprender e intervenir, y se sostiene la necesidad de resituar dicha comprensión en una dimensión más amplia, donde las políticas relacionadas, las construcciones culturales y las prácticas asociadas son percibidas como factores permanentes en la emergencia y recurrencia del ASI.

Por otro lado, entre los factores protectores señalados como importantes por ambas cohortes están: resolución de conflictos en forma adecuada, presencia de redes de apoyo para los/as integrantes de la familia, límites claros al interior de la familia, y relaciones democráticas que reconozcan al niño/a como sujeto de derecho. Se visualiza, entonces, la instalación de una perspectiva de mayor amplitud en el fenómeno del ASI, a pesar de no indicar distintas dimensiones entre los posibles factores protectores de esta problemática.

3.c. Dinámicas relacionales en el ASI: En ambas cohortes, los actores implicados en el ASI (abusador, víctima y/o figuras parentales como protectoras) no presentan un perfil característico, lo que marca la imposibilidad de realizar tipologías propias de la emergencia y mantención del ASI como fenómeno singularizado, aunque sí se hace referencia a ciertas características fundamentales, relativas a dinámicas relacionales intrafamiliares, que se encuentran de manera reiterada en mucha de la casuística del fenómeno.

4. Discurso institucional del ASI: A juicio de ambas cohortes, tanto las prácticas jurídicas como el cuerpo legal, configurados institucionalmente, establecen las bases para las líneas de intervención sobre el ASI, determinando incluso concepciones respecto de lo femenino y la infancia, en un discurso que se percibe limitado y discordante con la realidad misma del fenómeno. En este sentido, los dispositivos que la institucionalidad ofrece son, en el mejor de los casos, sensibles a las situaciones graves de violencia y abuso, pero ineficaces ante la violencia moderada. En esta misma lógica, lo que adquiere importancia son los marcos legales en los que se reproduce el discurso, el que es signado por la ideología hegemónica de nuestra sociedad, sin permitir la comprensión de la complejidad clínica con que el fenómeno ASI se inscribe en otras lógicas explicativas. 
4.a. Necesidad de interdisciplinariedad: Esta afirmación tuvo fuerza recurrente en ambas cohortes, lo que es coherente con el espíritu de las políticas institucionales que se plantean en los centros que atienden al ASI. Pese a la existencia de trabajo en equipo ampliado, los/as profesionales consideran insuficiente la cantidad de horas que cada centro dispone para reuniones clínicas, análisis de casos conjuntos y otros. Además, se remarca la necesidad de superar la falta de articulación entre lo que se demanda desde los procesos judiciales y las acciones especificas relativas a procesos terapéuticos, acciones preventivas e intervención psicosocial en general.

Por otra parte, en ambas cohortes se da cuenta de la prevalencia de prácticas donde impera la preocupación por los indicadores estadísticos en cuanto metas a alcanzar; como número de casos, término de procesos, datos sobre eficiencia y eficacia y otros, por sobre el abordaje singular y comprensivo de cada situación como fenómeno clínico, describiéndose un escenario donde coexisten focos divergentes en las dinámicas de trabajo: uno orientado hacia lo terapéutico y otro hacia las metas estadísticas.
Los propios terapeutas reconocen tener posturas conflictivas respecto de aquéllas promovidas por las instituciones, las que reproducen inconsistencias sobre los discursos interdisciplinarios para el afrontamiento del ASI.

4. b. Desasosiego y malestar subjetivo en profesionales que atienden ASI: En ambas cohortes hay malestares asociados a estilos y prácticas de trabajo. Tanto la escasa interdisciplinariedad, espacios para socializar las distintas perspectivas, divergencias de énfasis en lo micro y macro estadístico versus el proceso terapéutico, superposición entre miradas clínicas y/o jurídicas o institucionales y las dificultades en los alcances teóricos para abordar el fenómeno ASI en toda su magnitud. Ello promueven la impotencia de los profesionales para responder con eficacia los discursos dominantes, tanto de la sobrepatologización como los normativos jurídicos. Situaciones todas, en permanente tensión y conflicto en las aproximaciones de resolución clínica del fenómeno.

$1^{\text {era }}$ y $2^{\text {da }}$ Cohortes

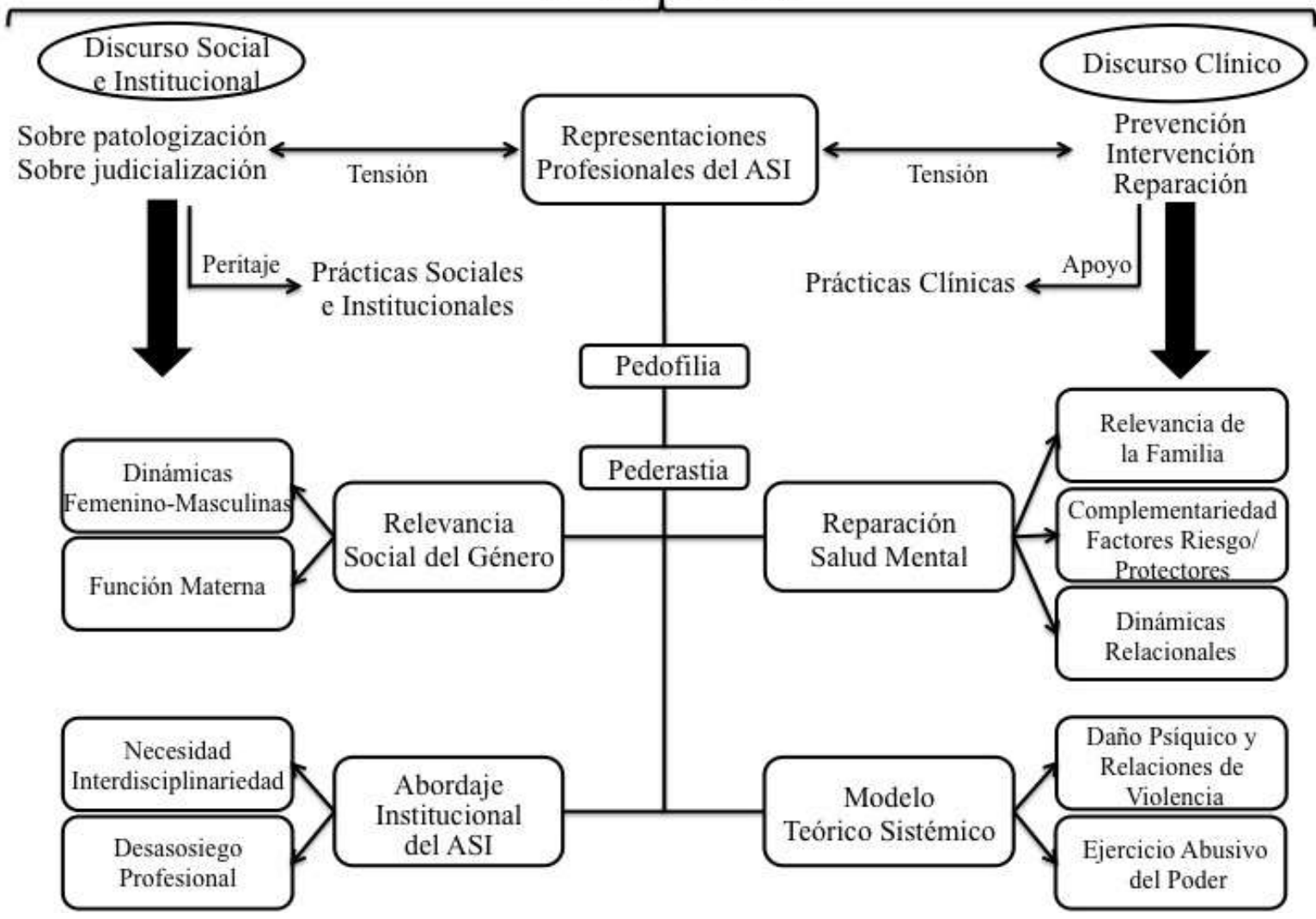

Figura 1. Representaciones de los profesionales acerca de la tensión en el abordaje e intervención del Abuso Sexual Infantil (ASI). 


\section{Discusión}

Desde los resultados se observa un escenario en que los profesionales que trabajan en el ámbito del abuso tienen consenso en conceptualizar al ASI como una forma de ejercer violencia por medio de la sexualidad en la dinámica familiar $\mathrm{y}$, por lo tanto, comprenderlo como una problemática abordable a partir de los referentes conceptuales desarrollados por los modelos sistémicos que se ocupan de la violencia al interior de la familia (VIF). No obstante, junto a estos argumentos (de carácter más bien empírico) a la hora de teorizar, se desliza una comprensión articulada en torno a las nociones de pedofilia y pederastia. De esta manera, la mirada de los/as profesionales es encauzada al campo de la psicopatología, dejando en un segundo plano aspectos que pueden ser inscritos en un contexto de comprensión más amplio que el de su morbidez.

En esta relación se superponen lecturas divergentes sobre un mismo fenómeno, cada una validada por distinto valor explicativo: por una parte se señala la recurrente presencia de dinámicas familiares con relaciones gravemente alteradas, y al mismo tiempo, persiste una aproximación hegemonizada por la marcación de los rasgos patológicos particulares del sujeto pederasta, que anteceden a su actuar y al contexto sociocultural en el cual ocurren, limitando la potencia clínica de la perspectiva teórica sistémica del espacio familiar, y marginando otras lecturas posibles, que permitan intervenir desde contextos más amplios. Existe, entonces, una limitación en el abordaje teórico determinada por la dificultad de lograr apreciaciones comprensivas amplias, que permitan inscribir conceptualmente al ASI de manera que intervenciones psicosociales y terapéuticas integradoras sitúen lo singular de cada situación en un marco referencial coherente a la envergadura psicosocial que articula la complejidad de la problemática, incluyendo todas sus singularidades.

En consecuencia, sólo las líneas de intervención derivadas de los planteamientos sistémicos son valoradas como el referente teórico/práctico más idóneo para la comprensión del ASI y, no obstante esto, su utilización incluso resulta limitada al focalizar su uso al espacio de la psicoterapia individual, dejando en segundo plano al familiar e ignorando totalmente al contextual amplio. Al mismo tiempo, prevalece la representación del pederasta y la pedofilia como figuras privilegiadas al momento de pensar en las causas del ASI, imponiéndose una mirada ligada al modelo médico tradicional de la psicopatología, que tiende a desvincular al abuso de las condiciones histórico sociales que cultivan su emergencia y permanencia.
De manera semejante, en la representación que los profesionales realizan de las relaciones intrafamiliares, se marca el ejercicio abusivo del poder como condición facilitada por la persistencia de creencias y patrones de convivencia transmitidos socialmente, y asociados a formas rígidas de significar los vínculos de poder al interior de la familia. A pesar de esta lectura, las intervenciones clínicas son orientadas principalmente a la reparación, circunscribiendo su acción al trabajo terapéutico con la víctima del abuso -siguiendo enfoques clínicos tradicionales centrados en la infancia, que ignoran contextos en dinámicas de poder- y en menor medida a las familias, sin integrar ni aprovechar los elementos del meso y macrosistema presentes en el ASI. En consecuencia, el ASI es retraducido y limitado a un fenómeno psicopatológico que oculta en esa representación la recurrencia existente entre el abuso, la transgresión de las relaciones familiares y el uso abusivo del poder. En síntesis, se trata al ASI desde el manejo clínico terapéutico coyuntural a las consecuencias del abuso, invisibilizando comprensiones esenciales que sólo pueden ser atendidas al alero de prácticas culturales ligadas a la dimensión de la violencia de género.

Aunque el ASI es inscrito en la visión de los profesionales como una forma de violencia, persiste la tensión entre su imagen como resultante de la patología individual del pederasta/perverso y aquella que nos remite a una representación del ASI como consecuencia de graves perturbaciones de las pautas relacionales al interior del orden familiar, dificultando una comprensión de las implicaciones de dicha problemática como una forma de violencia que interpela a lo social en su conjunto.

En este sentido, el escenario descrito en torno al ASI es el de un campo de comprensión donde los/as profesionales reconocen que la dimensión conflictiva del género encuadra una serie de condiciones culturales que sitúan la sexualidad y subjetividad femenina en el plano de la satisfacción del otro, antes que la propia, y en la dependencia y objetivación del deseo por parte de lo masculino. De esta forma, se revela la categoría del género presente a través de las manifestaciones y variaciones tanto de la VIF y del ASI, como dimensión articuladora de prácticas inscritas en el discurso hegemónico del machismo y la construcción de una feminidad delimitada y definida por este relato, y aún así, una reflexión que no decanta en prácticas terapéuticas concretas que respondan a la representación del ASI, como una forma de agresión atravesada por las características de la violencia de género.

Esta mirada sobre la incidencia del género en la emergencia y permanencia del ASI, orienta la representación de 
los profesionales en la comprensión de sus causas hacia un espacio más amplio que el familiar, de forma tal que es visto como una problemática con consecuencias que se proyectan al espacio social y que trasciende la esfera privada. Así, se considera que el daño asociado al abuso infantil repercute en la estabilidad de las instituciones y en los espacios públicos de convivencia, condicionando la perpetuación de algunas estrategias de afrontamiento y algunas pautas relacionales que seguirían sosteniendo acciones de violencia y abuso. En este sentido, en la representación de los profesionales, las construcciones de la masculinidad y feminidad imperantes en la cultura chilena permiten comprender la reproducción y mantención al interior del ASI, de tal que todos participaríamos de construcciones de género que otorgan sentido y legitiman dichos abusos.

Un caso particular pero relevante en la interacción entre lo público y lo privado de este fenómeno, es la figura de la madre y el rol materno. Los profesionales, al referirse a esta figura y su papel en el abuso, describen una gama variada de posibles respuestas, que van desde la "activación" del develamiento del secreto (pedir ayuda, abandonar el hogar cuando el abusador no lo hace, realizar la denuncia) hasta, en su contraparte, una mantención del secreto (desconociendo el reclamo del niño/a, omitiendo el abuso) e incluso, en un grado mayor, incentivando la situación de abuso en tanto se oculta el develamiento de los menores.

Al considerar las representaciones expresadas por los profesionales, la ambivalencia con que es concebido el rol materno en el origen y mantención del ASI se muestra análoga a la ambigüedad con que son señalados los factores socioculturales o psicológicos como causa única en la presencia de esta problemática. La mujer/madre es vista como agente pasivo del ASI, en cuanto es situada como sujeto subordinado a las construcciones hegemónicas del género, pero al mismo tiempo, es vista como agresora en cuanto la mirada se restringe a la mujer en particular, develando una tensión irreductible entre la representación de la madre como agente causa o facilitadora del abuso y la mujer determinada por ciertas construcciones sociales de lo femenino, de las cuales se nutre el rol materno, adoptando, por ende, las posiciones de víctima o victimaria.

Esta relación permanente entre las concomitantes públicas y privadas del ASI, adquiere relevancia en la visión de los profesionales cuando ésta dirige su mirada al contexto más amplio de la salud mental. Una impresión compartida por las distintas representaciones recabadas, descansa en reconocer que son las políticas jurídicas y las leyes derivadas de éstas las que articulan y dan forma a las intervenciones realizadas. En este escenario, existe la conciencia de que sin denuncia es imposible visibilizar la situación, y, por lo tanto, pensar en intervenciones mas integrales y efectivas.

En esta lógica, el trabajo de los profesionales y, el reconocimiento del ASI está condicionado a la necesaria retraducción del abuso a los términos emanados desde el discurso jurídico. Como resultado de esto, en la representación de los/as profesionales, el ASI como objeto de atención adquiere forma y es validado en la medida que dicha agresión constituye un delito tipificado y sancionable al interior del sistema jurídico. Por tal razón, el discurso de la salud mental queda subordinado a éste, que le otorga o resta legitimidad. Así, las visiones de lo femenino y la infancia son teñidas desde las políticas públicas y los marcos jurídicos establecidos.

En consecuencia, esta sobrejudicialización del tema tiene como efecto la obturación del discurso clínico en toda su complejidad, impidiendo su potencia comprensiva, generando una subordinación del discurso propio (psicológico) al discurso del otro (jurídico), dejando en entredicho el saber de la psicología en tanto lo que tiene que demostrar no es la coherencia de su explicación, sino, más bien, el grado de ajuste a la objetivación del fenómeno a la lógica delictual.

Además, los profesionales relatan una práctica tensionada por dos miradas conceptuales: una preocupada de dar cuenta del delito y establecer las condiciones del castigo (la que normativiza y externaliza la experiencia del abuso), y otra orientada a dar cabida a lo singular del sufrimiento puesto en juego (la que despliega aquello de particular en la experiencia de abuso), que es condición para crear lecturas comprensivas sobre la subjetividad de la experiencia, y por tanto, permitir las posibilidades de reparación, promoción, psicoterapia y salud mental. Dichos discursos conflictivos emanan de la pugna entre una cultura orientada hacia la identificación y sanción del abuso sexual, y otra cultura sensible a la necesidad de centrar el cuidado en el bienestar de los niños/as abusados y reparar en el espacio donde el abuso se ha perpetrado.

En esta lógica, la intervención orientada al abordaje judicial del ASI tendría un efecto de homogeneización sobre la comprensión de la situación, ya que la necesaria inscripción de los fenómenos, en los términos propios de los antecedentes delictuales, tiene el riesgo de invisibilizar lo singular de cada caso. Prevalece, entonces, una práctica discursiva donde impera lo estadístico en cuanto metas a alcanzar, por sobre la singularidad de cada situación como fenómeno clínico. Esta falta de coherencia discursiva propicia un escenario donde existen focos divergentes en las dinámicas de trabajo: uno orientado hacia lo terapéutico y otro hacia la penalización. 
Desde el discurso psicosocial, entonces, se agudiza la tensión entre operar hacia la intermediación técnica para la resolución judicial o, por el contrario, atender a la conflictiva del ASI, enmarcada en los parámetros consustanciales a la comprensión de la práctica clínica de la salud mental. Esta tensión, intrínsecamente contradictoria, cultiva una práctica incapaz de satisfacer tanto las miradas propias de lo psicológico, como la práctica consecuente a ellas, dejando al quehacer profesional propio de la salud mental, habitado por una desasosegante sensación de malestar.

Desde ahí parece importante la necesidad de abordar una perspectiva que trascienda los enfoques parcializados, tanto de la dimensión psicológica como de la dimensión normativo jurídica, indicando condiciones sociales estructurales y contextuales, develando sus dinámicas relacionales, y sus injerencias tanto en las causas como en la prevalencia del ASI. Así, el abuso nunca se articula en políticas de prevención ni intervención consistentes, quedando limitadas a la trinchera de la reparación. En relación a este análisis, además, se marca la congruencia producida en ambas cohortes sobre la necesidad de un trabajo interdisciplinario, que permita una perspectiva consistente con la complejidad característica del ASI.

Finalmente, es necesario apuntar que en el análisis de los discursos de los profesionales de ambas cohortes no se encuentran diferencias relevantes en las formas de representar y significar al ASI, salvo la distinción de algunas connotaciones que se resaltan más en una u otra cohorte, haciendo distintos énfasis ya sea en lo social o en lo intrafamiliar, como es el caso de aquello que se resalta respecto de la complementariedad de los factores de riesgo/factores protectores en nuestros resultados. De la misma manera, no se aprecian prácticas clínicas notoriamente distinguibles. Esto nos hace hipotetizar que, a pesar de la recurrencia y dramatismo con que este hecho social se nos presenta en Chile, no existen cambios sustantivos ni desarrollos profesionales significativos, ni en la comprensión ni en la intervención y/o reparación del ASI en toda su complejidad, al menos en estos últimos ocho años. Se instala, entonces, el desafío de investigar y proponer nuevas formas de aproximación a la problemática del ASI, que aborden eficaz y rigurosamente este polivalente fenómeno clínico.

\section{Referencias}

Asociación Chilena Pro Naciones Unidas (2010). Visión Bicentenario-Un Chile donde todos los niños y niñas conozcan sus derechos. Santiago: ACHNU.

Canela, R., Cea, N., Galindo G., y Valilla, S. (2010). Metodología de la investigación educativa: Investigación ex post facto. Universidad Autónoma de Madrid.

Echeburúa, E., y Guerricaechevarría, C. (2005) El Abuso sexual en la infancia: víctimas y agresores. Un enfoque clínico. Barcelona: Ariel.

Fresno, A., y Spencer, R. (2011). Efecto del Maltrato Físico en la Calidad de las Representaciones de Apego Infantil en Chile: Resultados Preliminares. Terapia Psicológica, 29, 191-196.

Gómez, E., Muñoz, M., y Haz, A. (2007). Familias multiproblemáticas y en riesgo social: características e intervención. Psykhe, 16, 43-54.

Gómez, E., y Haz, A. (2008). Intervención Familiar Preventiva en Programas Colaboradores del SENAME: La Perspectiva del Profesional. Psykhe [online], 17, 53-65. Recuperado desde <http://www. scielo.cl/scielo.php?script $=$ sci_arttextypid $=\mathrm{S} 0718222820080002$ $00005 y$ lng=esynrm=iso $>$. ISSN $0718-2228$. doi:10.4067/S071822282008000200005

Gonçalves, H. S., y Ferreira, A. L. (2002). A notificação da violência intrafamiliar contra crianças e adolescentes por profissionais da saúde. Caderno de Saúde Pública, 18, 315-319.

Habigzang, L. F., y Caminha, R. M. (2004). Abuso sexual contra crianças e adolescentes: Conceituação e intervenção clínica. São Paulo: Casa do Psicólogo.

Habizang, L., Koller, S., Azen Azevedo, G., y Machado, P. (2005). Abuso Sexual Infantil e Dinamica Familiar: Aspectos Observados em Procesos Juridicos. Psicología: Teoría e Pesquisa, 3, 341-348.

Herrero, A. (2009). Abuso sexual infantil. Revista de Epistemología y Ciencias Humanas, 1, 69-77.

Informe anual del Instituto Australiano de Salud y Bienestar Social, AIHW (2012). Protección de la Infancia 2010-2011. Recuperado desde http://www.juliecollins.fahcsia.gov.au/mediareleases/2012/pages/ jc m childprotection_20january2012.aspx

Instituto Nacional de Estadísticas (2003). Censo 2002. Resultados Población y Vivienda. Santiago: INE.

Lameiras-Fernández, M. (2002). Abusos sexuales en la Infancia: Abordaje Psicológico y Jurídico. Madrid: Biblioteca Nueva.

Martínez, M. (2006). La investigación cualitativa (síntesis conceptual). Revista IIPSI, 9, 123-146.

Morales, G., y Lira, E. (2000). La "receta" del autocuidado: Los riesgos de equipos en programas de trabajo con violencia. En O. Vilchez (Ed.), Violencia en la cultura: Riesgos y estrategias de intervención. Santiago: Ediciones Sociedad Chilena de Psicología Clínica.

Nelson, E., Heath, A., Madden, P. (2002). Association between self-reported childhood sexual abuse and adverse psychosocial outcomes: results from a twin study. En Archives of General Psychiatry, 59, 139- 145.

Renvoize, J. (1982). Incest: A Family History. Londres: Routledge y Kegan Paul.

UNICEF (2006). "Niños, niñas y adolescentes víctimas de delitos sexuales, en el marco de la reforma procesal penal". Recuperado desde http:// www.unicef.cl/unicef/public/archivos_documento/173/Informe $\% 20$ final.pdf 
